\title{
Dynamic Nonlinear Algebraic Models With Scale- Similarity Dynamic Procedure For Large-Eddy Simulation of Turbulence
}

\section{Zelong Yuan ( 11930910@mail.sustech.edu.cn )}

Southern University of Science and Technology https://orcid.org/0000-0003-0279-111X

\section{Yunpeng Wang}

Southern University of Science and Technology

\section{Chenyue Xie}

Southern University of Science and Technology

Jianchun Wang

Southern University of Science and Technology https://orcid.org/0000-0001-5101-7791

\section{Research Article}

Keywords: subgrid-scale model, nonlinear algebraic model, large-eddy simulation, incompressible turbulence

Posted Date: November 29th, 2021

DOI: https://doi.org/10.21203/rs.3.rs-1078014/v1

License: (c) (i) This work is licensed under a Creative Commons Attribution 4.0 International License. Read Full License

Version of Record: A version of this preprint was published at Advances in Aerodynamics on March 30th, 2022. See the published version at https://doi.org/10.1186/s42774-022-00107-z. 
Correspondence:

wangjc@sustech.edu.cn

${ }^{1}$ Department of Mechanics and Aerospace Engineering, Southern University of Science and Technology, Shenzhen, 518055 China

Full list of author information is available at the end of the article

\begin{abstract}
A dynamic nonlinear algebraic model with scale-similarity dynamic procedure (DNAM-SSD) is proposed for subgrid-scale (SGS) stress in large-eddy simulation of turbulence. The model coefficients of the DNAM-SSD model are adaptively calculated through the scale-similarity relation, which greatly simplifies the conventional Germano-identity based dynamic procedure (GID). The a priori study shows that the DNAM-SSD model predicts the SGS stress considerably better than the conventional velocity gradient model (VGM), dynamic Smagorinsky model (DSM), dynamic mixed model (DMM) and DNAM-GID model at a variety of filter widths ranging from inertial to viscous ranges. The correlation coefficients of the SGS stress predicted by the DNAM-SSD model can be larger than $95 \%$ with the relative errors lower than 30\%. In the a posteriori testings of LES, the DNAM-SSD model outperforms the implicit LES (ILES), DSM, DMM and DNAM-GID models without increasing computational costs, which only takes up half the time of the DNAM-GID model. The DNAM-SSD model accurately predicts plenty of turbulent statistics and instantaneous spatial structures in reasonable agreement with the filtered DNS data. These results indicate that the current DNAM-SSD model is attractive for the development of highly accurate SGS models for LES of turbulence.
\end{abstract}

Keywords: subgrid-scale model; nonlinear algebraic model; large-eddy simulation; incompressible turbulence

\section{Introduction}

Turbulent flows involve a wide range of length scales across several orders of magnitude, therefore the direct numerical simulation (DNS) of turbulence at high Reynolds number is impractical to solve all flow scales ranging from inertial to viscous ranges[1, 2, 3]. Large-eddy simulation (LES) is an effective approach which adopts the coarse mesh to merely resolve the large flow scales and model the effect of residual subgrid scales (SGS) on the resolved large scales[4, 5, 6, 7]. Extensive SGS models are proposed to reconstruct the unclosed SGS stress in previous works, including the Smagorinsky model[8, 9, 10], the velocity-gradient model (VGM)[11], the scale-similarity model[12, 13], the implicit LES (ILES) [14, 15, 16], the Reynolds-stress-constrained LES model[17], the datadriven models[18, 19, 20, 21, 22, 23, 24, 25], etc. The Smagorinsky model is one of the commonly-used SGS models whose model coefficient for the original version is statically adjusted by the experimental and DNS data in the early stage. 
Germano et al.[26] and Lilly[27] pioneered the development of a dynamical procedure based on the Germano identity through the least-squares algorithm, which makes the parameter of the dynamic Smagorinsky model (DSM) dynamically determined as the flow changes. Subsequently, the dynamical versions of some conventional SGS models with the Germano-identity based dynamic procedure (GID) were proposed $[4,5,6,7]$, including the dynamic mixed model (DMM) [28, 29, 30, 31], the dynamic Clark model[32], the dynamic localization model[33], etc.

The Smagorinsky model[8, 9, 26, 27] constructs the SGS stress with the linear constitutive relation based on the Boussinesq hypothesis, which requires the alignment between the SGS stress and the filtered strain-rate tensor. Pope[34] derived the general expression between the Reynolds stress and the averaged strain-rate and rotation-rate tensors with eleven integrity basis tensors based on the theory of invariants. Due to the expensive calculations of the high-order basis tensors in the general expression, the numerical verification of Pope's general viscous hypothesis was only limited to the two-dimensional turbulence[34]. Lund and Novikov[35] showed that the sixth invariant can be expressed as the ratio of the other five invariants, and reduced the original eleven polynomial basis tensors to five, which greatly simplified the computational complexity of the nonlinear algebraic SGS model in LES calculations. Especially, the anisotropic part of the SGS stress can be expressed as the general expression of the resolved strain-rate and rotation-rate tensors with five model coefficients[35]. Speziale et al.[36, 37] further simplified the Lund's general expression to a quadratic constitutive relation for the Reynolds stress. The model coefficients of the nonlinear algebraic model were mostly determined by the DNS data in the early research work. Wong[38] proposed a two-parameter dynamic nonlinear algebraic model (DNAM) using the quadratic constitutive relation with the Germano-identity based dynamic procedure. Kosović[39] applied the nonlinear constitutive relation to the shear-driven boundary layers at high Reynolds number. Wang et al.[40, 41] proposed a dynamic SGS model based on the quadratic nonlinear constitutive expression with local stability. Marstorp et al.[42] proposed an explicit algebraic SGS stress model with the equilibrium assumptions made on the partial-differential equations of SGS stress, and successfully applied to the rotational channel flow. Recently, a stochastic extension of the explicit algebraic SGS models has been developed by Rasam et al.[43]

In our previous research work, a nonlinear algebraic model based on the artificial neural network (ANN-NAM) was proposed[44], whose model coefficients are predicted by the invariant-input ANN with embedded invariance. The ANN-NAM model[44] reconstructs the SGS stress and statistics of velocity with high accuracy both in the a priori and a posteriori analyses of LES. Wang et al.[45] proposed a ANN-based semi-explicit spatial gradient model with embedded invariance. A dynamic version of the spatial gradient model (DSGM)[46] was proposed for the parameter determination strategy. Yuan et al. developed deconvolutional artificial neural network (DANN)[21] and dynamic iterative approximate deconvolution (DIAD) models[22] to recover the local unfiltered velocity with the neighboring spatial stencils of the filtered velocity. A scale-similarity-based dynamic procedure (SSD) was proposed to adaptively calculate the weights of the spatial stencil[22]. The DIAD model with the SSD procedure is superior to the other conventional dynamic SGS 
models in the reconstruction of the statistics of velocity and transient coherent structures of turbulence[22].

In the current research, a novel dynamic nonlinear algebraic model with scalesimilarity dynamic procedure (DNAM-SSD) is developed for reconstructing the unclosed SGS stress in LES of incompressible turbulence. The performance of the DNAM-SSD model is examined by comparing with those of some classical SGS models both in the a priori and a posteriori testings of LES at two filter widths $\bar{\Delta}=16 h_{\mathrm{DNS}}$ and $32 h_{\mathrm{DNS}}$ with the corresponding grid resolutions of $N=128^{3}$ and $64^{3}$. The computational accuracy and costs of the newly-proposed scale-similarity based dynamic (SSD) procedure are compared to the conventional Germano-identity based dynamic (GID) procedure. The remainder of the paper is organized as follows. The governing equations of LES will be described in Section 2. The introductions of the conventional SGS models and DNAM models are respectively illustrated in Sections 3 and 4 . Sections 5 will conduct the numerical simulation of incompressible isotropic turbulence. The a priori and a posteriori studies are correspondingly provided in Sections 6 and 7. Conclusions are drawn in Section 8.

\section{Governing equations of large-eddy simulation}

The incompressible turbulence is governed by the Navier-Stokes equations, whose dimensionless conservation form is written as[1]

$$
\begin{aligned}
& \frac{\partial u_{i}}{\partial x_{i}}=0, \\
& \frac{\partial u_{i}}{\partial t}+\frac{\partial\left(u_{i} u_{j}\right)}{\partial x_{j}}=-\frac{\partial p}{\partial x_{i}}+\frac{1}{\operatorname{Re}} \frac{\partial^{2} u_{i}}{\partial x_{j} \partial x_{j}}+\mathcal{F}_{i},
\end{aligned}
$$

where $u_{i}$ denotes the $i$-th velocity component $(i=1,2,3$ represents the three directions of the Cartesian coordinate system, respectively.), $p$ is the pressure, Re is the Reynolds number, and $\mathcal{F}_{i}$ is the $i$-th large-scale force component.[47, 48, 21] For brevity and simplicity, we adopt the summation convection for the repeated indices by default in this paper.

Besides, the governing dimensionless parameter for the incompressible turbulence, namely, the Taylor microscale Reynolds number $R e_{\lambda}$ is given by[1]

$$
R e_{\lambda}=\frac{u^{\mathrm{rms}} \lambda}{\sqrt{3} \nu}
$$

where $\nu$ denotes the kinematic viscosity and $u^{\mathrm{rms}}=\sqrt{\left\langle u_{i} u_{i}\right\rangle}$ is the root-mean-square $(\mathrm{rms})$ value of the velocity magnitude. Here, " $\bullet \bullet "$ represents a spatial average over the entire computational domain. In addition, the Taylor microscale $\lambda$ is expressed as[1]

$$
\lambda=u^{\mathrm{rms}} \sqrt{5 \nu / \varepsilon},
$$

where $\varepsilon=2 \nu\left\langle S_{i j} S_{i j}\right\rangle$ denotes the dissipation rate and $S_{i j}=\frac{1}{2}\left(\partial u_{i} / \partial x_{j}+\partial u_{j} / \partial x_{i}\right)$ is the strain-rate tensor. 
For the large-eddy simulation, the resolved large scales are separated from the subgrid small scales by the spatial filtering operation, which is introduced as[1,2]

$$
\bar{f}(\mathbf{x})=\int_{\Omega} f(\mathbf{x}-\mathbf{r}) G(\mathbf{r} ; \bar{\Delta}) d \mathbf{r},
$$

where $f(\mathbf{x})$ represents the arbitrary physical variable, and an overbar stands for the low-pass spatial filtering. Here, $\Omega$ denotes the entire physical domain, with $G$ and $\bar{\Delta}$ respectively being the spatial filter function and filter width. The governing equations for the LES can be obtained by applying the spatial filtering on the Eqs. (1) and (2), correspondingly, which can be derived as[1, 2]

$$
\begin{aligned}
& \frac{\partial \bar{u}_{i}}{\partial x_{i}}=0, \\
& \frac{\partial \bar{u}_{i}}{\partial t}+\frac{\partial\left(\bar{u}_{i} \bar{u}_{j}\right)}{\partial x_{j}}=-\frac{\partial \bar{p}}{\partial x_{i}}-\frac{\partial \tau_{i j}}{\partial x_{j}}+\frac{1}{\operatorname{Re}} \frac{\partial^{2} \bar{u}_{i}}{\partial x_{j} \partial x_{j}}+\overline{\mathcal{F}}_{i} .
\end{aligned}
$$

Here, the unclosed SGS stress $\tau_{i j}$ in the Eq. (7) is defined by $[4,5,6]$

$$
\tau_{i j}=\overline{u_{i} u_{j}}-\bar{u}_{i} \bar{u}_{j}
$$

The SGS stress involves the nonlinear interactions between the resolved large scales and under-solved small scales, therefore additional SGS stress modeling is required to close the governing equations of LES. In the following two sections, the conventional SGS models and the proposed dynamic nonlinear algebraic models with scale-similarity dynamic procedure (SSD) are respectively described for the LES computations.

\section{Conventional SGS models}

The explicit modeling for the unclosed SGS stress can be divided into the functional modeling and structural modeling. The functional models mimic the forward energy transfer from the resolved large scales to the residual small scales by constructing the explicit eddy-viscosity forms, while the structural modeling is established by the truncated series expansions or the hypothesis of scale similarity to correctly recover the SGS stress with high accuracy. A typical functional model is the dynamic Smagorinsky model (DSM), whose constitutive relation for the deviatoric SGS stress is given by $[26,27]$

$$
\tau_{i j}^{A}=\tau_{i j}-\frac{\delta_{i j}}{3} \tau_{k k}=-2 C_{S}^{2} \bar{\Delta}^{2}|\bar{S}| \bar{S}_{i j}
$$

where $|\bar{S}|=\left(2 \bar{S}_{i j} \bar{S}_{i j}\right)^{1 / 2}$ is the characteristic filtered strain rate, and $\bar{S}_{i j}=$ $\frac{1}{2}\left(\partial \bar{u}_{i} / \partial x_{j}+\partial \bar{u}_{j} / \partial x_{i}\right)$ is the filtered strain-rate tensor. The superscript "A" represents the trace-free part of the arbitrary variables, namely, $(\bullet)_{i j}^{A}=(\bullet)_{i j}-(\bullet)_{k k} \delta_{i j} / 3$. Here, the isotropic SGS stress $\tau_{k k}$ is absorbed into the pressure term. $C_{S}^{2}$ is the 
Smagorinsky coefficient, which can be determined by the Germano identity dynamic procedure (GID). The test-filter level SGS stress with the double-filtering scale $\tilde{\bar{\Delta}}=2 \bar{\Delta}$ is expressed as $[26,27]$

$$
\mathcal{T}_{i j}=\widetilde{\overline{u_{i} u_{j}}}-\tilde{\bar{u}}_{i} \tilde{\bar{u}}_{j}
$$

where a tilde stands for the test filtering operation at the filter scale $\tilde{\bar{\Delta}}$. The deviatoric part of $\mathcal{T}_{i j}$ can be modeled based on the scale-invariance hypothesis, defined by $[26,27]$

$$
\mathcal{T}_{i j}^{A}=\mathcal{T}_{i j}-\frac{\delta_{i j}}{3} \mathcal{T}_{k k}=-2 C_{S}^{2} \tilde{\bar{\Delta}}^{2}\left|\tilde{\bar{S}}^{\mid}\right| \tilde{\bar{S}}_{i j}
$$

These two SGS stresses with different filter scales, namely, $\tau_{i j}$ and $\mathcal{T}_{i j}$ satisfy the Germano identity, expressed as[26]

$$
\mathcal{L}_{i j}=\mathcal{T}_{i j}-\tilde{\tau}_{i j}=\widetilde{\bar{u}_{i} \bar{u}_{j}}-\tilde{\bar{u}}_{i} \tilde{\bar{u}}_{j}
$$

where the Leonard stress $\mathcal{L}_{i j}$ can be calculated by the resolved filtered field for LES calculations. Therefore, the optimal Smagorinsky coefficient $C_{S}^{2}$ can be further determined by the least-squares algorithm, namely[27]

$$
C_{S}^{2}=\frac{\left\langle\mathcal{L}_{i j}^{A} \mathcal{M}_{i j}\right\rangle}{\left\langle\mathcal{M}_{k l} \mathcal{M}_{k l}\right\rangle}
$$

where $\mathcal{L}_{i j}^{A}=\mathcal{L}_{i j}-\frac{1}{3} \delta_{i j} \mathcal{L}_{k k}$, and $\mathcal{M}_{i j}=\tilde{\alpha}_{i j}-\beta_{i j}$. Here $\alpha_{i j}=2 \bar{\Delta}^{2}|\bar{S}| \bar{S}_{i j}, \beta_{i j}=$ $2 \tilde{\bar{\Delta}}^{2} \mid \tilde{\bar{S}}_{\mid} \tilde{\bar{S}}_{i j}$

A typical structural model is the velocity gradient model (VGM) based on the truncated Taylor series expansions, given by[11]

$$
\tau_{i j}=\frac{\bar{\Delta}^{2}}{12} \frac{\partial \bar{u}_{i}}{\partial x_{k}} \frac{\partial \bar{u}_{j}}{\partial x_{k}}
$$

The dynamic mixed model (DMM) combines the scale-similarity model with the dissipative Smagorinsky term, which can overcome the deficiency of numerical instability in the structural modeling of the SGS stress. The SGS stresses constructed by the DMM model at scales $\bar{\Delta}$ and $\tilde{\bar{\Delta}}$ are expressed, respectively, as[49, 12, 28]

$$
\begin{gathered}
\tau_{i j}=C_{1} h_{1, i j}+C_{2} h_{2, i j}, \\
\mathcal{T}_{i j}=C_{1} H_{1, i j}+C_{2} H_{2, i j},
\end{gathered}
$$

where $h_{1, i j}=-2 \bar{\Delta}^{2}|\bar{S}| \bar{S}_{i j}, h_{2, i j}=\widetilde{\bar{u}}_{i} \overline{\bar{u}}_{j}-\tilde{\bar{u}}_{i} \tilde{\bar{u}}_{j}, H_{1, i j}=-2 \tilde{\bar{\Delta}}^{2}|\tilde{\bar{S}}| \tilde{\bar{S}}_{i j}$, and $H_{2, i j}=$ $\widehat{\overline{\tilde{u}}}_{i} \widehat{\overline{\tilde{u}}}_{j}-\hat{\tilde{\bar{u}}}_{i} \hat{\bar{u}}_{j}$. Here, the hat stands for the test filtering at scale $\hat{\Delta}=4 \bar{\Delta}$. Similar to 
the DSM model, the model coefficients $C_{1}$ and $C_{2}$ are calculated by the Germano identity dynamic procedure, namely[21, 22]

$$
\begin{aligned}
& C_{1}=\frac{\left\langle N_{i j}^{2}\right\rangle\left\langle\mathcal{L}_{i j} M_{i j}\right\rangle-\left\langle M_{i j} N_{i j}\right\rangle\left\langle\mathcal{L}_{i j} N_{i j}\right\rangle}{\left\langle N_{i j}^{2}\right\rangle\left\langle M_{i j}^{2}\right\rangle-\left\langle M_{i j} N_{i j}\right\rangle^{2}}, \\
& C_{2}=\frac{\left\langle M_{i j}^{2}\right\rangle\left\langle\mathcal{L}_{i j} N_{i j}\right\rangle-\left\langle M_{i j} N_{i j}\right\rangle\left\langle\mathcal{L}_{i j} M_{i j}\right\rangle}{\left\langle N_{i j}^{2}\right\rangle\left\langle M_{i j}^{2}\right\rangle-\left\langle M_{i j} N_{i j}\right\rangle^{2}},
\end{aligned}
$$

where $M_{i j}=H_{1, i j}-\tilde{h}_{1, i j}$, and $N_{i j}=H_{2, i j}-\tilde{h}_{2, i j}$.

\section{Dynamic nonlinear algebraic models (DNAM)}

In the SGS stress modeling, the constitute relation of the unclosed SGS stress can be regarded as the function of the local filtered quantities, i.e., the filtered strain-rate tensor $\bar{S}_{i j}$ and filtered rotation-rate tensor $\bar{\Omega}_{i j}$, namely[34, 35]

$$
\tau_{i j}=f\left(\bar{S}_{i j}, \bar{\Omega}_{i j} ; \delta_{i j}, \bar{\Delta}\right)
$$

where the filtered rotation-rate tensor $\bar{\Omega}_{i j}=\frac{1}{2}\left(\partial \bar{u}_{i} / \partial x_{j}-\partial \bar{u}_{j} / \partial x_{i}\right)$. For brevity and simplicity of the tensorial polynomials, the matrix multiplications for the tensor contractions are expressed as[34, 35, 40]

$$
\overline{\mathbf{S}}^{2}=\bar{S}_{i k} \bar{S}_{k j}, \quad \overline{\mathbf{S}} \overline{\mathbf{\Omega}}=\bar{S}_{i k} \bar{\Omega}_{k j}, \operatorname{tr}\left(\overline{\mathbf{S}} \overline{\mathbf{\Omega}}^{2}\right)=\bar{S}_{i j} \bar{\Omega}_{j k} \bar{\Omega}_{k i} .
$$

A general expression of the modeled SGS stress [Eq. (19)] can be expanded to the sum of an infinite number of tensorial polynomials with the form $\overline{\mathbf{S}}^{m_{1}} \overline{\mathbf{\Omega}}^{n_{1}} \overline{\mathbf{S}}^{m_{2}} \overline{\mathbf{\Omega}}^{n_{2}} \ldots$, where $m_{i}$ and $n_{i}$ are positive integers. The infinite tensorial polynomials can be reduced to a finite number by the Cayley-Hamilton theorem, thus the modeled SGS stress is expressed as the linear combination of the basis tensors formed by the product of $\overline{\mathbf{S}}$ and $\overline{\mathbf{\Omega}}$, namely[34]

$$
\tau_{i j}=\sum_{n=1}^{11} g_{n}\left(\lambda_{1}, \lambda_{2}, \cdots, \lambda_{6}\right) T_{i j}^{(n)},
$$

where $T_{i j}^{(n)}$ is the $n$-th basis tensor and the model coefficients $g_{n}$ are functions of the six integrity invariants $\lambda_{m}(m=1,2, \cdots, 6)$. Here, the eleven basis tensors $T_{i j}^{(n)}$ and six independent invariants $\lambda_{m}$ are respectively expressed as[34]

$$
\begin{aligned}
& \mathbf{T}^{(1)}=\overline{\mathbf{S}}, \quad \mathbf{T}^{(2)}=\overline{\mathbf{S}}^{2}, \\
& \mathbf{T}^{(3)}=\overline{\mathbf{\Omega}}^{2}, \quad \mathbf{T}^{(4)}=\overline{\mathbf{S}} \overline{\boldsymbol{\Omega}}-\overline{\boldsymbol{\Omega}} \overline{\mathbf{S}}, \\
& \mathbf{T}^{(5)}=\overline{\mathbf{S}}^{2} \overline{\boldsymbol{\Omega}}-\overline{\boldsymbol{\Omega}} \overline{\mathbf{S}}^{2}, \quad \mathbf{T}^{(6)}=\mathbf{I}, \\
& \mathbf{T}^{(7)}=\overline{\mathbf{S}} \overline{\boldsymbol{\Omega}}^{2}+\overline{\mathbf{\Omega}}^{2} \overline{\mathbf{S}}, \quad \mathbf{T}^{(8)}=\overline{\mathbf{\Omega}} \overline{\mathbf{S}} \overline{\boldsymbol{\Omega}}^{2}-\overline{\mathbf{\Omega}}^{2} \overline{\mathbf{S}} \overline{\boldsymbol{\Omega}}, \\
& \mathbf{T}^{(9)}=\overline{\mathbf{S}} \overline{\boldsymbol{\Omega}} \overline{\mathbf{S}}^{2}-\overline{\mathbf{S}}^{2} \overline{\boldsymbol{\Omega}} \overline{\mathbf{S}}, \mathbf{T}^{(10)}=\overline{\mathbf{S}}^{2} \overline{\boldsymbol{\Omega}}^{2}+\overline{\mathbf{\Omega}}^{2} \overline{\mathbf{S}}^{2}, \\
& \mathbf{T}^{(11)}=\overline{\boldsymbol{\Omega}} \overline{\mathbf{S}}^{2} \overline{\boldsymbol{\Omega}}^{2}-\overline{\boldsymbol{\Omega}}^{2} \overline{\mathbf{S}}^{2} \overline{\boldsymbol{\Omega}},
\end{aligned}
$$




$$
\begin{array}{ll}
\lambda_{1}=\operatorname{tr}\left(\overline{\mathbf{S}}^{2}\right), & \lambda_{2}=\operatorname{tr}\left(\overline{\mathbf{\Omega}}^{2}\right), \\
\lambda_{3}=\operatorname{tr}\left(\overline{\mathbf{S}}^{3}\right), & \lambda_{4}=\operatorname{tr}\left(\overline{\mathbf{S}} \overline{\mathbf{\Omega}}^{2}\right), \\
\lambda_{5}=\operatorname{tr}\left(\overline{\mathbf{S}}^{2} \overline{\mathbf{\Omega}}^{2}\right), & \lambda_{6}=\operatorname{tr}\left(\overline{\mathbf{S}}^{2} \overline{\mathbf{\Omega}}^{2} \overline{\mathbf{S}} \overline{\mathbf{\Omega}}\right) .
\end{array}
$$

If the model coefficients $g_{n}$ are relaxed as the ratios of polynomials of these integrity invariants, the number of the above basis tensors can be reduced from eleven to five. In accordance with the dimensional consistency, the anisotropic part of the modeled SGS stress can be given by[35]

$$
\begin{aligned}
\tau_{i j}^{A} & =\bar{\Delta}^{2} \sum_{n=1}^{5} C_{n} \mathbb{T}_{i j}^{(n), A} \\
& =\bar{\Delta}^{2}\left[C_{1}|\overline{\mathbf{S}}| \overline{\mathbf{S}}+C_{2}\left(\overline{\mathbf{S}}^{2}\right)^{A}+C_{3}\left(\overline{\mathbf{\Omega}}^{2}\right)^{A}+C_{4}(\overline{\mathbf{S}} \overline{\boldsymbol{\Omega}}-\overline{\mathbf{\Omega}} \overline{\mathbf{S}})+\frac{C_{5}}{|\overline{\mathbf{S}}|}\left(\overline{\mathbf{S}}^{2} \overline{\mathbf{\Omega}}-\overline{\mathbf{\Omega}} \overline{\mathbf{S}}^{2}\right)\right]
\end{aligned}
$$

where the characteristic filtered strain rate $|\overline{\mathbf{S}}|=\left(2 \bar{S}_{i j} \bar{S}_{i j}\right)^{1 / 2}$, and $C_{n}$ are five dimensionless model coefficients. The corresponding basis tensors $\mathbb{T}_{i j}^{(n)}$ that satisfy the consistent dimension with the square of the velocity gradient are defined by

$$
\mathbb{T}_{i j}^{(1)}=|\overline{\mathbf{S}}| \overline{\mathbf{S}}, \mathbb{T}_{i j}^{(2)}=\overline{\mathbf{S}}^{2}, \mathbb{T}_{i j}^{(3)}=\overline{\mathbf{\Omega}}^{2}, \mathbb{T}_{i j}^{(4)}=\overline{\mathbf{S}} \overline{\boldsymbol{\Omega}}-\overline{\mathbf{\Omega}} \overline{\mathbf{S}}, \quad \mathbb{T}_{i j}^{(5)}=\frac{1}{|\overline{\mathbf{S}}|}\left(\overline{\mathbf{S}}^{2} \overline{\mathbf{\Omega}}-\overline{\mathbf{\Omega}} \overline{\mathbf{S}}^{2}\right)
$$

In the paper, two dynamic procedures are adopted to determine the model coefficients $C_{n}$ of the dynamic nonlinear algebraic models (DNAM). One is the Germano identity dynamic (GID) procedure based on the scale-invariance assumption, and the other is newly proposed scale-similarity dynamic (SSD) procedure in accordance with the scale-similarity relation. The rest of this section will be divided into two subsections to respectively introduce these two different modeling approaches.

\subsection{DNAM models with Germano identity dynamic procedure (DNAM-GID)}

Similar to the conventional dynamic SGS models (e.g. DSM and DMM models), the DNAM model with Germano identity dynamic procedure, abbreviated as DNAMGID, introduces the test-filter level SGS stress $\mathcal{T}_{i j}$ at the double-filtering scale $\tilde{\bar{\Delta}}=$ $2 \bar{\Delta}$, modeled by

$$
\begin{aligned}
\mathcal{T}_{i j}^{A} & =\tilde{\bar{\Delta}}^{2} \sum_{n=1}^{5} C_{n} \mathbb{H}_{i j}^{(n), A} \\
& =\tilde{\bar{\Delta}}^{2}\left[C_{1}|\tilde{\overline{\mathbf{S}}}| \tilde{\overline{\mathbf{S}}}+C_{2}\left(\tilde{\overline{\mathbf{S}}}^{2}\right)^{A}+C_{3}\left(\tilde{\overline{\mathbf{\Omega}}}^{2}\right)^{A}+C_{4}(\tilde{\overline{\mathbf{S}}} \tilde{\overline{\mathbf{\Omega}}}-\tilde{\overline{\mathbf{\Omega}}} \tilde{\overline{\mathbf{S}}})+\frac{C_{5}}{|\tilde{\overline{\mathbf{S}}}|}\left(\tilde{\overline{\mathbf{S}}}^{2} \tilde{\overline{\mathbf{\Omega}}}-\tilde{\overline{\mathbf{\Omega}}}^{2}\right)\right] .
\end{aligned}
$$


Here, $\mathbb{H}_{i j}^{(n)}$ is the $n$-th basis tensor at the test filter scale $\tilde{\bar{\Delta}}=2 \bar{\Delta}$, expressed as

$\mathbb{H}_{i j}^{(1)}=|\tilde{\overline{\mathbf{S}}}| \tilde{\overline{\mathbf{S}}}, \mathbb{H}_{i j}^{(2)}=\tilde{\overline{\mathbf{S}}}^{2}, \mathbb{H}_{i j}^{(3)}=\tilde{\overline{\mathbf{\Omega}}}^{2}, \mathbb{H}_{i j}^{(4)}=\tilde{\overline{\mathbf{S}}} \tilde{\bar{\Omega}}-\tilde{\overline{\mathbf{\Omega}}} \tilde{\overline{\mathbf{S}}}, \quad \mathbb{H}_{i j}^{(5)}=\frac{1}{|\tilde{\overline{\mathbf{S}}}|}\left(\tilde{\overline{\mathbf{S}}}^{2} \tilde{\overline{\mathbf{\Omega}}}-\tilde{\overline{\mathbf{\Omega}}} \tilde{\overline{\mathbf{S}}}^{2}\right)$

Consistent with Eq. (12), the modeled SGS stresses $\tau_{i j}$ and $\mathcal{T}_{i j}$ at different filter scales satisfy the Germano identity, namely

$$
\mathcal{L}_{i j}^{A}=\mathcal{T}_{i j}^{A}-\tilde{\tau}_{i j}^{A}=\sum_{n=1}^{5} C_{n} \mathbb{M}_{i j}^{(n)}
$$

where $\mathbb{M}_{i j}^{(n)}=\tilde{\bar{\Delta}}^{2} \mathbb{H}_{i j}^{(n), A}-\bar{\Delta}^{2} \tilde{\mathbb{T}}_{i j}^{(n), A}$. The model coefficients $C_{n}$ can be further calculated by the least-squares algorithm, derived by

$$
\sum_{m=1}^{5} C_{m}\left\langle\mathbb{M}_{i j}^{(m)} \mathbb{M}_{i j}^{(n)}\right\rangle=\left\langle\mathcal{L}_{i j}^{A} \mathbb{M}_{i j}^{(n)}\right\rangle, \quad(n=1,2, \cdots, 5)
$$

For the DNAM-GID model, the optimal model coefficients $C_{n}$ can be obtained by solving the system of five linear equations in Eq. (29).

\subsection{DNAM models with scale-similarity dynamic procedure (DNAM-SSD)}

In this paper, we propose a novel scale-similarity dynamic procedure for the DNAM model to determine the optimal model coefficients dynamically. The real SGS stress can be regarded as the nonlinear function of the velocity $u_{i}$ and the filter kernel at scale $\bar{\Delta}$, whereas the SGS stress modeled by the the DNAM model has the nonlinear constitutive relation with the local filtered physical quantities (e.g. the filtered strain-rate and rotation-rate tensors $\overline{\mathbf{S}}$ and $\overline{\boldsymbol{\Omega}}$ ),

$$
\begin{aligned}
\tau_{i j}^{A} & =\tau_{i j}^{A}\left(u_{i} ; \bar{\Delta}\right)=\left(\overline{u_{i} u_{j}}-\bar{u}_{i} \bar{u}_{j}\right)^{A}=\sum_{n=1}^{5} C_{n} \mathbb{T}_{i j}^{(n), A}(\overline{\mathbf{S}}, \overline{\mathbf{\Omega}}) \\
& =\bar{\Delta}^{2}\left[C_{1}|\overline{\mathbf{S}}| \overline{\mathbf{S}}+C_{2}\left(\overline{\mathbf{S}}^{2}\right)^{A}+C_{3}\left(\overline{\mathbf{\Omega}}^{2}\right)^{A}+C_{4}(\overline{\mathbf{S}} \overline{\boldsymbol{\Omega}}-\overline{\mathbf{\Omega}} \overline{\mathbf{S}})+\frac{C_{5}}{|\overline{\mathbf{S}}|}\left(\overline{\mathbf{S}}^{2} \overline{\mathbf{\Omega}}-\overline{\mathbf{\Omega}} \overline{\mathbf{S}}^{2}\right)\right]
\end{aligned}
$$

Based on the scale-similarity hypothesis, the modeled SGS stress at the filter scale $\tilde{\Delta}$ shares the consistent model coefficients $C_{n}$ with that at the filter scale $\bar{\Delta}$, namely

$$
\begin{aligned}
\tau_{i j}^{A}\left(u_{i} ; \tilde{\Delta}\right) & =\left(\widetilde{u_{i} u_{j}}-\tilde{u}_{i} \tilde{u}_{j}\right)^{A}=\sum_{n=1}^{5} C_{n} \mathbb{T}_{i j}^{(n), A}(\tilde{\mathbf{S}}, \tilde{\mathbf{\Omega}}) \\
& =\tilde{\Delta}^{2}\left[C_{1}|\tilde{\mathbf{S}}| \tilde{\mathbf{S}}+C_{2}\left(\tilde{\mathbf{S}}^{2}\right)^{A}+C_{3}\left(\tilde{\boldsymbol{\Omega}}^{2}\right)^{A}+C_{4}(\tilde{\mathbf{S}} \tilde{\boldsymbol{\Omega}}-\tilde{\mathbf{\Omega}} \tilde{\mathbf{S}})+\frac{C_{5}}{|\tilde{\mathbf{S}}|}\left(\tilde{\mathbf{S}}^{2} \tilde{\boldsymbol{\Omega}}-\tilde{\mathbf{\Omega}} \tilde{\mathbf{S}}^{2}\right)\right]
\end{aligned}
$$


The constitutive equation of the SGS stress is assumed to be invariant to the physical field, therefore we can replace the unfiltered velocity $u_{i}$ with the filtered velocity $\bar{u}_{i}$ in Eq. (30) and obtain

$$
\begin{aligned}
\mathcal{L}_{i j}^{A} & =\tau_{i j}^{A}\left(\bar{u}_{i} ; \tilde{\Delta}\right)=\left(\widetilde{\bar{u}}_{i} \bar{u}_{j}-\tilde{\bar{u}}_{i} \tilde{\bar{u}}_{j}\right)^{A}=\sum_{n=1}^{5} C_{n} \mathbb{T}_{i j}^{(n), A}(\tilde{\overline{\mathbf{S}}}, \tilde{\overline{\mathbf{\Omega}}}) \\
& =\tilde{\Delta}^{2}\left[C_{1}|\tilde{\overline{\mathbf{S}}}| \tilde{\overline{\mathbf{S}}}+C_{2}\left(\tilde{\overline{\mathbf{S}}}^{2}\right)^{A}+C_{3}\left(\tilde{\overline{\mathbf{\Omega}}}^{2}\right)^{A}+C_{4}(\tilde{\overline{\mathbf{S}}} \tilde{\overline{\mathbf{\Omega}}}-\tilde{\overline{\mathbf{\Omega}}} \tilde{\overline{\mathbf{S}}})+\frac{C_{5}}{|\tilde{\overline{\mathbf{S}}}|}\left(\tilde{\overline{\mathbf{S}}}^{2} \tilde{\overline{\mathbf{\Omega}}}-\tilde{\overline{\mathbf{\Omega}}}_{\overline{\overline{\mathbf{S}}}}^{2}\right)\right] .
\end{aligned}
$$

$\mathcal{L}_{i j}^{A}$ is the anisotropic part of the resolved Leonard stress. For simplicity, we let $\mathbb{N}_{i j}^{(n)}=\mathbb{T}_{i j}^{(n), A}(\tilde{\overline{\mathbf{S}}}, \tilde{\overline{\mathbf{\Omega}}})$ and Eq. (32) is abbreviated as $\mathcal{L}_{i j}^{A}=\sum_{n=1}^{5} C_{n} \mathbb{N}_{i j}^{(n)}$. Since $\mathcal{L}_{i j}^{A}$ and $\mathbb{N}_{i j}^{(n)}$ are both resolved in the filtered field, the model coefficients $C_{n}$ can be determined by the least-squares method,

$$
\sum_{m=1}^{5} C_{m}\left\langle\mathbb{N}_{i j}^{(m)} \mathbb{N}_{i j}^{(n)}\right\rangle=\left\langle\mathcal{L}_{i j}^{A} \mathbb{N}_{i j}^{(n)}\right\rangle, \quad(n=1,2, \cdots, 5) .
$$

It is worth noting that the DNAM-SSD model only calculates $\mathbb{T}_{i j}^{(n), A}(\overline{\mathbf{S}}, \overline{\mathbf{\Omega}})$ and $\mathbb{T}_{i j}^{(n), A}(\tilde{\overline{\mathbf{S}}}, \tilde{\overline{\mathbf{\Omega}}})$ rather than $\mathbb{T}_{i j}^{(n), A}(\overline{\mathbf{S}}, \overline{\mathbf{\Omega}}), \mathbb{T}_{i j}^{(n), A}(\tilde{\overline{\mathbf{S}}}, \tilde{\overline{\mathbf{\Omega}}})$ and $\tilde{\mathbb{T}}_{i j}^{(n), A}(\overline{\mathbf{S}}, \overline{\mathbf{\Omega}})$ in the DNAM-GID model, therefore the scale-similarity dynamic procedure simplifies the conventional dynamic procedure based on the Germano identity. Besides, in the following sections, we can show that the DNAM model with the proposed scalesimilarity dynamic procedure performs better than that with the conventional GID procedure both in the a priori and the a posteriori testings of incompressible turbulence.

\section{Numerical simulation of incompressible isotropic turbulence}

In order to validate the performance of the proposed DNAM-SSD model, the numerical simulation of incompressible isotropic turbulence is performed in a cubic box of $(2 \pi)^{3}$ with periodic boundary conditions at the Taylor Reynolds number $R e_{\lambda} \approx 250$. The pseudo-spectral approach with the two-thirds dealiasing rule is adopted for the spatial discretization of the governing equation. A second-order explicit AdamsBashforth scheme[50] is applied to the time advancement. The large-scale forcing is implemented on the two lowest wavenumber shells[48, 47, 21] to keep the turbulence in equilibrium. In the paper, we use $N=1024^{3}$ uniform grids in the DNS calculation with the grid spacing $h_{\mathrm{DNS}}=2 \pi / 1024$. The kinematic viscosity is set to $\nu=1 / R e=0.001$. The detailed one-point statistics of DNS calculation are summarized in Table 1. Here, $k_{\max }=\frac{2 \pi}{3 h_{\mathrm{DNS}}}$ represents the largest effective wavenumber after the fully two-thirds dealiasing, and $\omega^{\mathrm{rms}}=\sqrt{\left\langle\omega_{i} \omega_{i}\right\rangle}$ stands for the root-meansquare value of the vorticity magnitude. The Kolmogorov length scale $\eta$ and the 
integral length scale $L_{I}$ represent the smallest resolved scale and the largest characteristic scale, which are defined, respectively, by

$$
\begin{aligned}
& \eta=\left(\frac{\nu^{3}}{\varepsilon}\right)^{1 / 4}, \\
& L_{I}=\frac{3 \pi}{2\left(u^{\mathrm{rms}}\right)^{2}} \int_{0}^{+\infty} \frac{E(k)}{k} d k,
\end{aligned}
$$

where $\varepsilon=2 \nu\left\langle S_{i j} S_{i j}\right\rangle$ is the dissipation rate. The total turbulent kinetic energy $E_{k}=\frac{1}{2}\left\langle u_{i} u_{i}\right\rangle=\int_{0}^{+\infty} E(k) d k$, and $E(k)$ stands for the velocity spectrum. The resolution parameter $k_{\max } \eta \geq 2.1$ is found to be sufficient enough for the convergence of turbulent kinetic energy at all scales[51, 52].

In the paper, the filtered physical quantities and the real SGS stress $\tau_{i j}$ are calculated by a Gaussian filter, which is expressed as[1,2]

$$
G(\mathbf{r} ; \bar{\Delta})=\left(\frac{6}{\pi \bar{\Delta}^{2}}\right)^{1 / 2} \exp \left(-\frac{6 \mathbf{r}^{2}}{\bar{\Delta}^{2}}\right)
$$

We select two filter scales $\left(\bar{\Delta}=16 h_{\mathrm{DNS}}\right.$ and $\left.32 h_{\mathrm{DNS}}\right)$ for model verification, and the corresponding cutoff wavenumbers are $k_{c}=\pi / \bar{\Delta}=32$ and 16 , respectively. Figure 1 shows the velocity spectra of the DNS and filtered DNS at both filter widths $\left(\bar{\Delta}=16 h_{\mathrm{DNS}}\right.$ and $\left.32 h_{\mathrm{DNS}}\right)$. The filtered velocity spectra almost overlap with the DNS data at the low-wavenumber region satisfying the Kolmogorov $k^{-5 / 3}$ scaling, while generally diminish with the increasing of wavenumbers, and drop rapidly at the region larger than the truncated wavenumber $k_{c}$. More kinetic energy is filtered out at a lager filter scale, therefore the filtered velocity spectrum at $\bar{\Delta}=32 h_{\text {DNS }}$ is lower than that at $\bar{\Delta}=16 h_{\mathrm{DNS}}$. Overall $95 \%$ and $88 \%$ of the turbulent kinetic energy are retained in the filtered velocity field at the filter widths $\bar{\Delta}=16 h_{\mathrm{DNS}}$ and $32 h_{\mathrm{DNS}}$, respectively.

Table 1: One-point statistics for the DNS with grid resolution of $1024^{3}$.

\begin{tabular}{ccccccccc}
\hline$R e_{\lambda}$ & $E_{k}$ & $k_{\max } \eta$ & $\eta / h_{\text {DNS }}$ & $L_{I} / \eta$ & $\lambda / \eta$ & $u^{\text {rms }}$ & $\omega^{\text {rms }}$ & $\varepsilon$ \\
\hline 252 & 2.63 & 2.11 & 1.01 & 235.2 & 31.2 & 2.30 & 26.90 & 0.73 \\
\hline
\end{tabular}

\section{A priori study of the DNAM Models}

In the a priori analysis, twenty snapshots of DNS data at equal temporal intervals during two large-eddy turnover periods $\left(\tau=L_{I} / u^{\text {rms }}\right)$ are adopted to examine the model accuracy of the DNAM-GID and DNAM-SSD models with several filter scales ranging from $\bar{\Delta}=4 h_{\mathrm{DNS}}$ to $\bar{\Delta}=64 h_{\mathrm{DNS}}$. Two evaluation metrics are used to quantify the distinction between the real value $\left(Q^{\text {real }}\right)$ and the modeled value $\left(Q^{\text {model }}\right)$ for targeted variable $Q$, namely the correlation coefficient $C(Q)$ and the relative error $E_{r}(Q)$, respectively defined by[21, 22]

$$
C(Q)=\frac{\left\langle\left(Q^{\text {real }}-\left\langle Q^{\text {real }}\right\rangle\right)\left(Q^{\text {model }}-\left\langle Q^{\text {model }}\right\rangle\right)\right\rangle}{\left\langle\left(Q^{\text {real }}-\left\langle Q^{\text {real }}\right\rangle\right)^{2}\right\rangle^{1 / 2}\left\langle\left(Q^{\text {model }}-\left\langle Q^{\text {model }}\right\rangle\right)^{2}\right\rangle^{1 / 2}},
$$




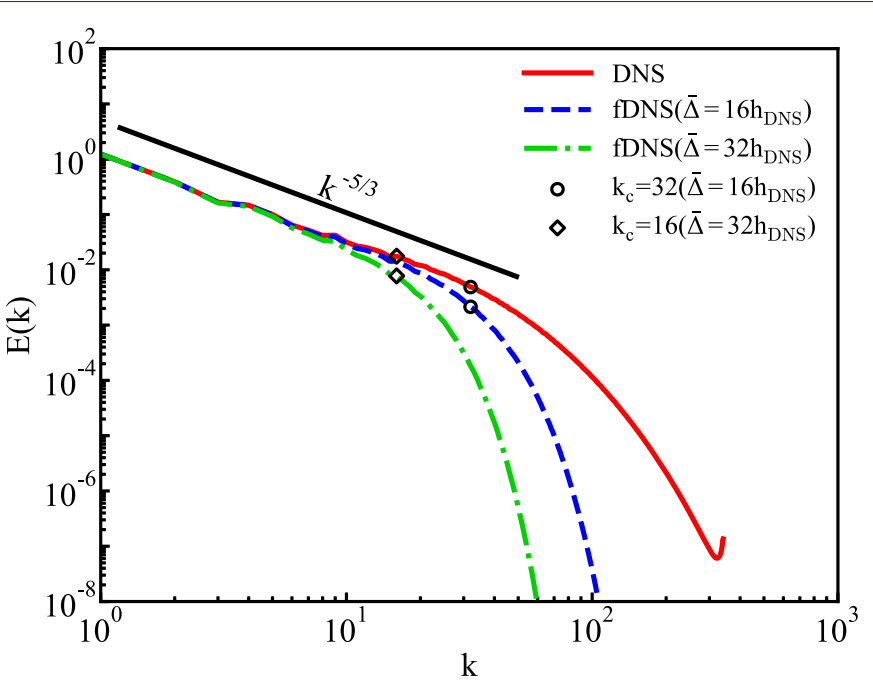

Figure 1: Velocity spectra of DNS and filtered DNS for incompressible isotropic turbulence $\left(N=1024^{3}\right)$.

where $\langle\bullet\rangle$ represents the ensemble average of total samples. In the a priori study, we first investigate the impact of integrity basis tensors $\mathbb{T}_{i j}^{(n), A}$ on the SGS stress $\tau_{i j}$ by calculating the correlation coefficients at different filter widths, shown in Fig. 2. The normal components of the basis tensors $\mathbb{T}_{11}^{(n), A}$ share the similar correlation coefficients $C\left(\mathbb{T}_{i j}^{(n), A}, \tau_{i j}^{A}\right)$ with those of shear components $\mathbb{T}_{12}^{(n), A}$. The fourth term $\mathbb{T}_{i j}^{(4), A}=\overline{\mathbf{S}} \overline{\boldsymbol{\Omega}}-\overline{\boldsymbol{\Omega}} \overline{\mathbf{S}}$ contributes the highest correlation coefficients about $80 \%$ with the SGS stress, while the first term $\mathbb{T}_{i j}^{(1), A}=|\overline{\mathbf{S}}| \overline{\mathbf{S}}$ gives the worst predictions with correlation coefficients lower than $30 \%$ among these five basis tensors. It is worth noting that the terms $\mathbb{T}_{i j}^{(3), A}=\left(\overline{\mathbf{\Omega}}^{2}\right)^{A}$ and $\mathbb{T}_{i j}^{(5), A}=\left(\overline{\mathbf{S}}^{2} \overline{\boldsymbol{\Omega}}-\overline{\boldsymbol{\Omega}} \overline{\mathbf{S}}^{2}\right) /|\overline{\mathbf{S}}|$ have higher correlation coefficients with the SGS stress compared to the second term $\mathbb{T}_{i j}^{(2), A}=\left(\overline{\mathbf{S}}^{2}\right)^{A}$, therefore we keep all five basis tensors without any simplification in the paper. With the increasing of the filter widths, the correlation coefficients between the first basis tensor $\mathbb{T}_{i j}^{(1), A}$ and the SGS stress constantly increase, while those of the other four terms gradually drop but still higher than those of the first term. These results indicate that the classical Smagorinsky model (linear relation with only the first basis tensor $\mathbb{T}_{i j}^{(1), A}$ ) cannot fully reconstruct the SGS stress.

Figures 2 and 3 respectively illustrate the correlation coefficients and relative errors of the normal and shear components of the SGS stress for different SGS models at a number of filter scales ranging from the inertial region to the dissipation range. Here, the VGM model is the velocity gradient model (see Eq. 14) which has high a priori accuracy among the classical SGS models. The DNAMLS model is a DNAM model with a priori knowledge of DNS data, whose model 
coefficients are calculated by the least-squares method using the real SGS stress, namely $\sum_{m=1}^{5} C_{m}\left\langle\mathbb{T}_{i j}^{(m), A} \mathbb{T}_{i j}^{(n), A}\right\rangle=\left\langle\tau_{i j}^{A} \mathbb{T}_{i j}^{(n), A}\right\rangle, \quad(n=1,2, \cdots, 5)$. The model coefficients of the DNAM-GID model and DNAM-SSD model are calculated by the dynamic procedure based on conventional Germano identity (cf. Eq. 29) and the newly proposed scale-similarity dynamic procedure (cf. Eq. 33), respectively.

The DNAM-LS model has the highest correlation coefficients and the lowest relative errors with the SGS stress, since the DNS data are used to determine the model coefficients. The correlation coefficients and relative errors predicted by the proposed DNAM-SSD model are very close to the DNAM-LS model at all filter scales, which are much better than the DNAM-GID and VGM models. The DNAM-SSD model predicts the SGS stress accurately with the correlation coefficients overall higher than $92 \%$ and the relative errors less than $40 \%$ ranging from the viscous region to the inertial region. In contrast, the DNAM-GID model gives the worst prediction among these SGS models with the relative errors approximately over $40 \%$. It is worth noting that the DNAM-SSD model performs better than the conventional VGM model at all filter widths, indicating that the basis tensors of the DNAM model are more complete than the velocity gradient in reconstructing the SGS stress.
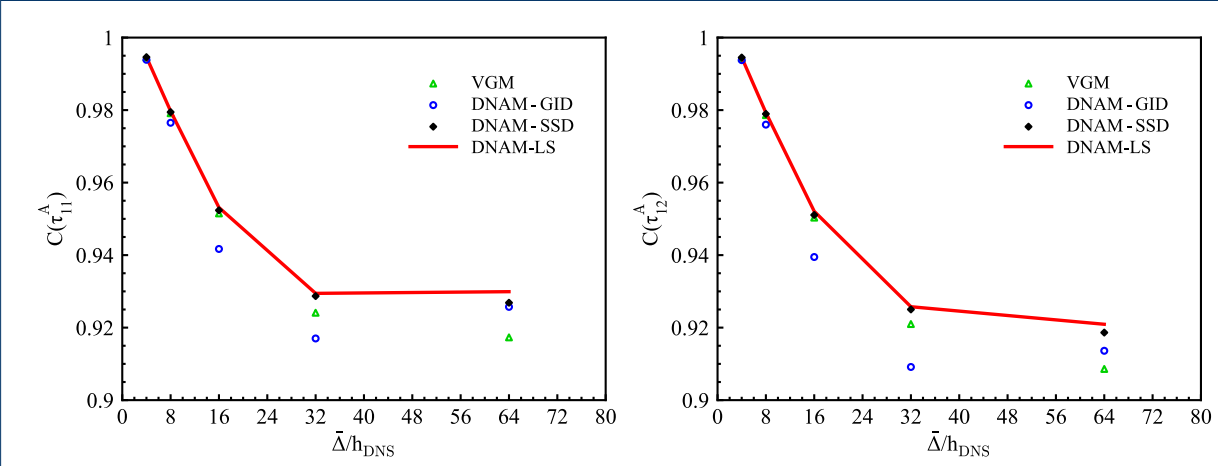

Figure 2: Correlation coefficients of normal and shear SGS stresses in a priori study.
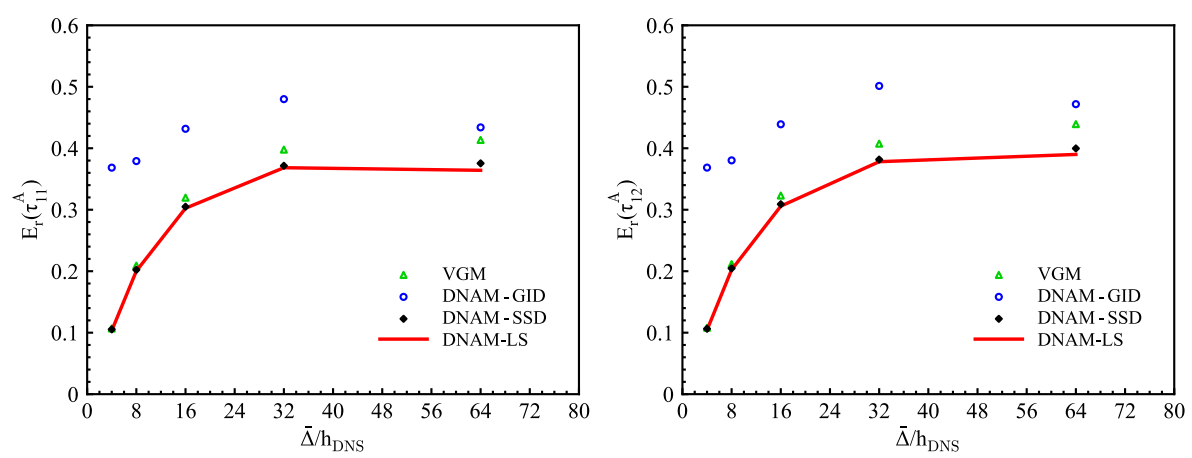

Figure 3: Relative errors of normal and shear SGS stresses in a priori study. 


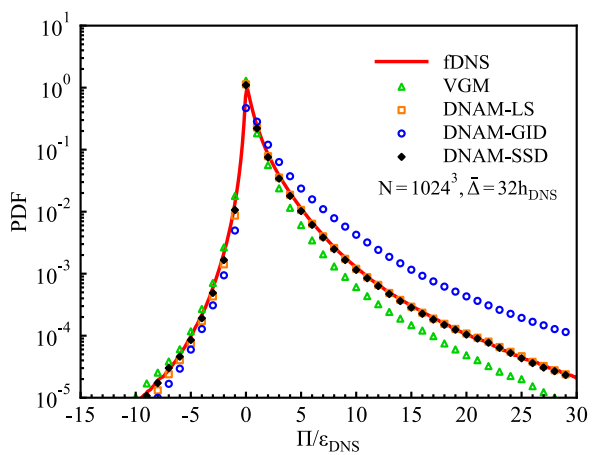

Figure 4: PDFs of normalized SGS energy flux $\Pi / \epsilon_{\mathrm{DNS}}$ in a priori study.

In order to further quantify the model accuracy of different SGS models in the $a$ priori analysis, we compare the correlation coefficients and relative errors of the SGS stress at filter widths $\bar{\Delta}=16 h_{\mathrm{DNS}}$ and $32 h_{\mathrm{DNS}}$ listed in Tables 3 and 4 , respectively. The DSM and DMM models use the Germano-identity dynamic procedure (see Eqs. 13, 17 and 18) to dynamically determine model coefficients. The DNAM-SSD and DNAM-LS models give the best prediction of the SGS stress with correlation coefficients higher than $95 \%$ and $92 \%$ as well as relative errors lower than $30 \%$ and $38 \%$ at corresponding filter widths $\bar{\Delta}=16 h_{\text {DNS }}$ and $32 h_{\text {DNS }}$ among these SGS models. In contrast, the DSM model performs the worst compared to other SGS models at both filter scales, whose correlation coefficients are lower than $30 \%$ and relative errors are nearly $100 \%$. The DNAM-GID model predicts the SGS stress obviously worse than the DNAM-SSD and DNAM-LS models, but still much better than the classical DMM model with the consistent GID dynamic procedure. Besides, the performance of the VGM model in a priori study is between the DNAM-GID model and DNAM-SSD model at both filter widths. These results demonstrate that the basis tensors of the DNAM model are more complete in modeling the SGS stress compared to those of the DMM model and VGM model. In the a priori analysis, the proposed scale-similarity dynamic procedure (SSD) shows distinct advantages over the conventional Germano-identity dynamic procedure (GID) in determining the model coefficients of SGS models.

Finally, we evaluate the SGS energy transfer for different SGS models by comparing the normalized SGS energy flux $\Pi / \epsilon_{\mathrm{DNS}}$, where $\Pi=-\tau_{i j} \bar{S}_{i j}$ represents the SGS energy flux and $\epsilon_{\mathrm{DNS}}$ denotes the dissipation rate calculated by the DNS data. The PDFs of the SGS energy flux reconstructed by the DNAM-SSD and DNAM-LS models coincide with the filtered DNS data at both filter scales $\bar{\Delta}=16 h_{\mathrm{DNS}}$ and $32 h_{\text {DNS }}$, which are obviously better than the VGM and DNAM-GID models. In comparison, DNAM-GID model fails to predict the SGS energy transfer, indicating that model-coefficient determination by the proposed SSD procedure is superior to that of the conventional GID procedure, and is well approximated to the DNAM-LS model in the a priori study.

\section{A posteriori study of the DNAM Models}

The a posteriori testing of LES is important to illustrate the practical performance of the SGS models. In LES computations, the kinematic viscosity $\nu=0.001$ is 
Table 2: Correlation coefficients $C\left(\mathbb{T}_{i j}^{(n), A}, \tau_{i j}^{A}\right)$ with the increasing of the filter scales.

\begin{tabular}{ccccccccccc}
\hline $\bar{\Delta} / h_{\text {DNS }}$ & $\mathbb{T}_{11}^{(1)}$ & $\mathbb{T}_{11}^{(2)}$ & $\mathbb{T}_{11}^{(3)}$ & $\mathbb{T}_{11}^{(4)}$ & $\mathbb{T}_{11}^{(5)}$ & $\mathbb{T}_{12}^{(1)}$ & $\mathbb{T}_{12}^{(2)}$ & $\mathbb{T}_{12}^{(3)}$ & $\mathbb{T}_{12}^{(4)}$ & $\mathbb{T}_{12}^{(5)}$ \\
\hline 2 & 0.227 & 0.44 & 0.518 & 0.824 & 0.549 & 0.219 & 0.429 & 0.513 & 0.819 & 0.545 \\
4 & 0.227 & 0.425 & 0.519 & 0.821 & 0.534 & 0.221 & 0.415 & 0.514 & 0.816 & 0.529 \\
8 & 0.233 & 0.401 & 0.513 & 0.808 & 0.509 & 0.228 & 0.394 & 0.509 & 0.804 & 0.5 \\
16 & 0.256 & 0.384 & 0.498 & 0.789 & 0.488 & 0.253 & 0.38 & 0.498 & 0.781 & 0.468 \\
32 & 0.3 & 0.385 & 0.487 & 0.777 & 0.491 & 0.306 & 0.389 & 0.493 & 0.755 & 0.448 \\
64 & 0.334 & 0.398 & 0.498 & 0.788 & 0.535 & 0.384 & 0.431 & 0.508 & 0.737 & 0.464 \\
\hline
\end{tabular}

Table 3: Correlation coefficients and relative errors of the SGS stress at filter scale $\bar{\Delta}=16 h_{\mathrm{DNS}}$ in the a priori study.

\begin{tabular}{lcccccc}
\hline Model $\backslash C\left(\tau_{i j}\right)$ & $\tau_{11}$ & $\tau_{22}$ & $\tau_{33}$ & $\tau_{12}$ & $\tau_{23}$ & $\tau_{13}$ \\
\hline DSM & 0.256 & 0.255 & 0.24 & 0.26 & 0.279 & 0.253 \\
DMM & 0.628 & 0.617 & 0.605 & 0.608 & 0.624 & 0.615 \\
VGM & 0.952 & 0.949 & 0.949 & 0.947 & 0.95 & 0.95 \\
DNAM-LS & 0.953 & 0.951 & 0.951 & 0.949 & 0.952 & 0.952 \\
DNAM-GID & 0.942 & 0.939 & 0.938 & 0.936 & 0.94 & 0.939 \\
DNAM-SSD & 0.952 & 0.95 & 0.95 & 0.948 & 0.951 & 0.951 \\
\hline Model $\backslash E_{r}\left(\tau_{i j}\right)$ & $\tau_{11}$ & $\tau_{22}$ & $\tau_{33}$ & $\tau_{12}$ & $\tau_{23}$ & $\tau_{13}$ \\
\hline DSM & 0.986 & 0.99 & 0.999 & 0.991 & 0.979 & 0.993 \\
DMM & 0.779 & 0.788 & 0.798 & 0.796 & 0.784 & 0.791 \\
VGM & 0.32 & 0.327 & 0.327 & 0.332 & 0.324 & 0.323 \\
DNAM-LS & 0.302 & 0.309 & 0.31 & 0.314 & 0.307 & 0.306 \\
DNAM-GID & 0.432 & 0.438 & 0.442 & 0.446 & 0.44 & 0.439 \\
DNAM-SSD & 0.305 & 0.312 & 0.313 & 0.318 & 0.31 & 0.309 \\
\hline
\end{tabular}

Table 4: Correlation coefficients and relative errors of the SGS stress at filter scale $\bar{\Delta}=32 h_{\mathrm{DNS}}$ in the a priori study.

\begin{tabular}{lcccccc}
\hline Model $\backslash C\left(\tau_{i j}\right)$ & $\tau_{11}$ & $\tau_{22}$ & $\tau_{33}$ & $\tau_{12}$ & $\tau_{23}$ & $\tau_{13}$ \\
\hline DSM & 0.294 & 0.29 & 0.295 & 0.325 & 0.308 & 0.32 \\
DMM & 0.6 & 0.597 & 0.596 & 0.61 & 0.602 & 0.609 \\
VGM & 0.922 & 0.921 & 0.92 & 0.922 & 0.921 & 0.922 \\
DNAM-LS & 0.927 & 0.926 & 0.925 & 0.927 & 0.926 & 0.927 \\
DNAM-GID & 0.913 & 0.913 & 0.912 & 0.913 & 0.913 & 0.914 \\
DNAM-SSD & 0.926 & 0.925 & 0.925 & 0.926 & 0.925 & 0.927 \\
\hline Model $\backslash E_{r}\left(\tau_{i j}\right)$ & $\tau_{11}$ & $\tau_{22}$ & $\tau_{33}$ & $\tau_{12}$ & $\tau_{23}$ & $\tau_{13}$ \\
\hline DSM & 0.975 & 0.978 & 0.977 & 0.963 & 0.971 & 0.964 \\
DMM & 0.802 & 0.804 & 0.806 & 0.797 & 0.803 & 0.797 \\
VGM & 0.405 & 0.407 & 0.409 & 0.405 & 0.407 & 0.404 \\
DNAM-LS & 0.376 & 0.377 & 0.379 & 0.375 & 0.378 & 0.374 \\
DNAM-GID & 0.479 & 0.479 & 0.482 & 0.483 & 0.483 & 0.48 \\
DNAM-SSD & 0.38 & 0.381 & 0.383 & 0.379 & 0.382 & 0.378 \\
\hline
\end{tabular}

Table 5: The computational cost of SGS stress modeling $\tau_{i j}$ for LES computations with the filter scales $\bar{\Delta}=16 h_{\text {DNS }}$ and $32 h_{\text {DNS }}$.

\begin{tabular}{lcccc}
\hline Model $\left(\Delta=16 h_{\text {DNS }}\right)$ & DSM & DMM & DNAM-GID & DNAM-SSD \\
\hline $\mathrm{t}(\mathrm{CPU} \cdot \mathrm{s})$ & 5.9 & 9.941 & 10.996 & 5.989 \\
$\mathrm{t} / \mathrm{t}_{\mathrm{DMM}}$ & 0.593 & 1 & 1.106 & 0.603 \\
Model $\left(\bar{\Delta}=32 h_{\text {DNS }}\right)$ & DSM & DMM & DNAM-GID & DNAM-SSD \\
\hline $\mathrm{t}(\mathrm{CPU} \cdot \mathrm{s})$ & 0.875 & 1.438 & 1.64 & 0.837 \\
$\mathrm{t} / \mathrm{t}_{\mathrm{DMM}}$ & 0.609 & 1 & 1.141 & 0.582 \\
\hline
\end{tabular}




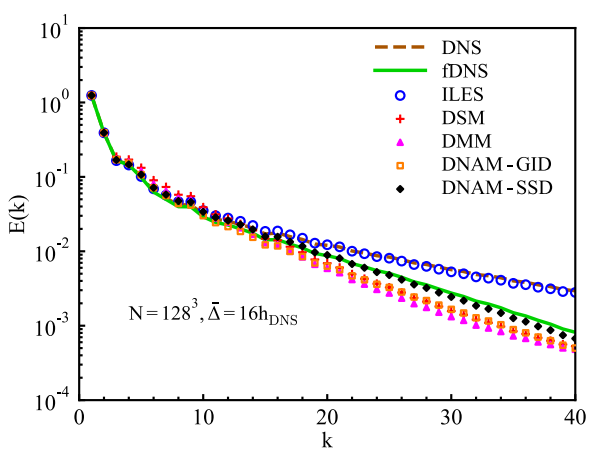

Figure 5: Comparisons of velocity spectra in a posteriori study at filter scale $\bar{\Delta}=16 h_{\mathrm{DNS}}$.
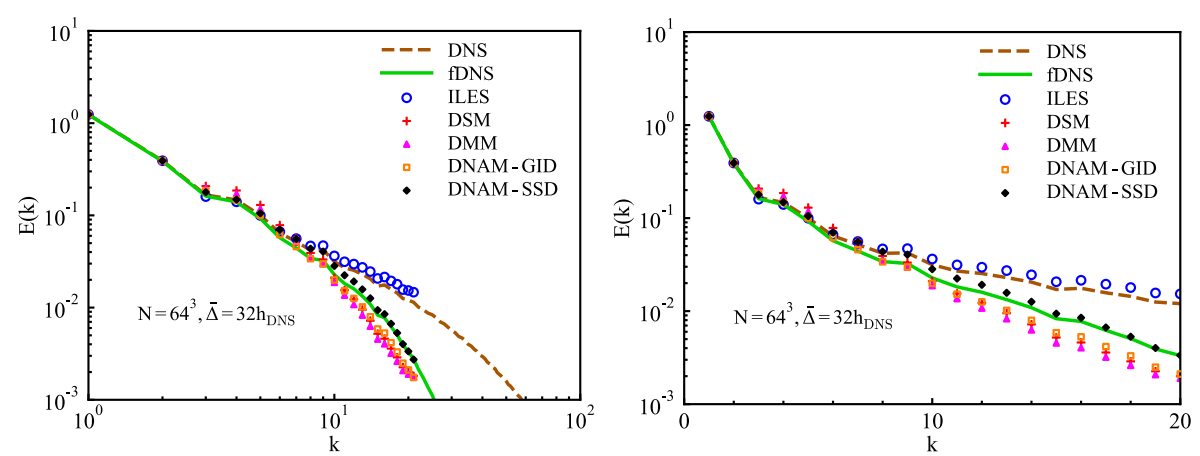

Figure 6: Comparisons of velocity spectra in a posteriori study at filter scale $\bar{\Delta}=32 h_{\mathrm{DNS}}$.
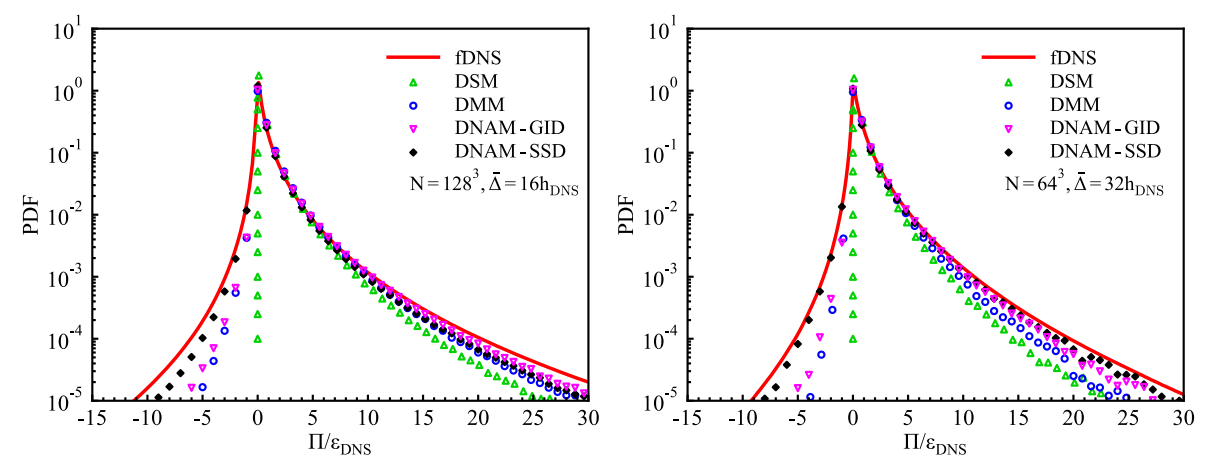

Figure 7: PDFs of the normalized SGS energy flux $\Pi / \epsilon_{\mathrm{DNS}}$ in a posteriori study.

consistent with that of DNS. Two filter scales $\bar{\Delta}=16 h_{\mathrm{DNS}}$ and $32 h_{\mathrm{DNS}}$ are selected to study the impact of filter widths on the SGS modeling. The newly proposed DNAM-SSD model is compared to the classical SGS models, including the implicit LES (ILES), the dynamic Smagorinsky model (DSM), the dynamic mixed model (DMM) and the dynamic nonlinear algebraic model with conventional Germanoidentity dynamic procedure (DNAM-GID). It has been found that the filter-to- 


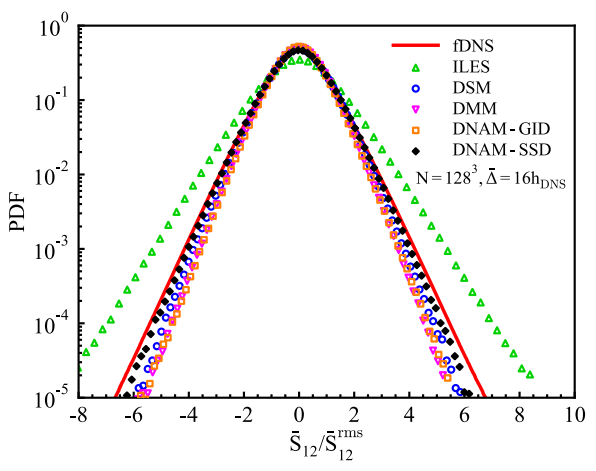

Figure 8: PDFs of the normalized strain-rate tensor in a posteriori study at filter scale $\bar{\Delta}=16 h_{\mathrm{DNS}}$.
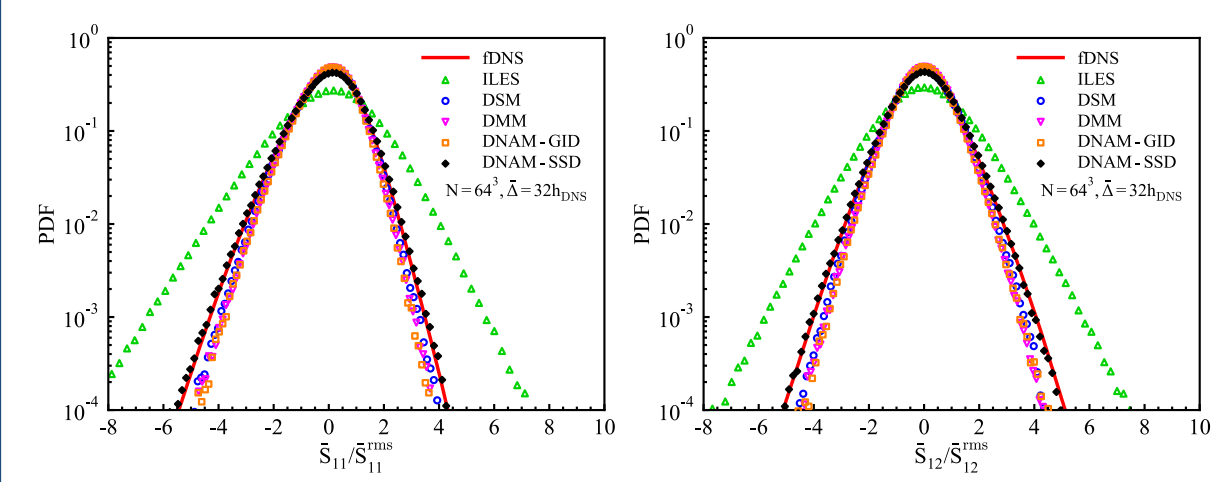

Figure 9: PDFs of the normalized strain-rate tensor in a posteriori study at filter scale $\bar{\Delta}=32 h_{\mathrm{DNS}}$.
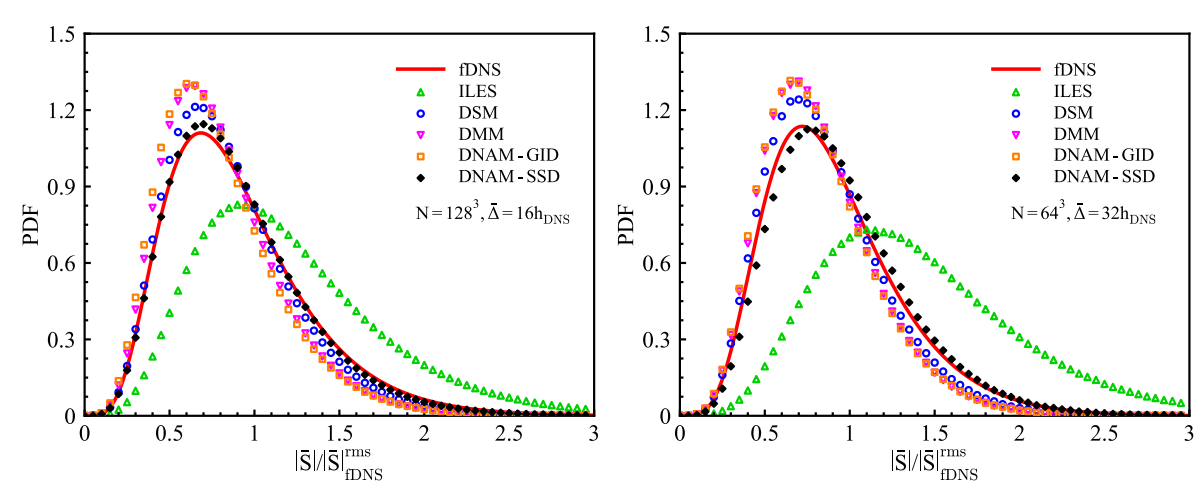

Figure 10: PDFs of the normalized characteristic strain rate in a posteriori study.

grid ratio $F G R=\bar{\Delta} / h_{\mathrm{LES}}=2$ can effectively reduce the influence of the spatial discretization errors on the SGS stress modeling[53, 54, 55]. Therefore, we fix the FGR value to 2 and the corresponding grid points of LES are $N=128^{3}$ and $64^{3}$ for the selected filter widths $\bar{\Delta}=16 h_{\mathrm{DNS}}$ and $32 h_{\mathrm{DNS}}$. It is worth noting that both filter scales lie in the inertial range (cf. Fig. 1) and the scale-invariance assumption 

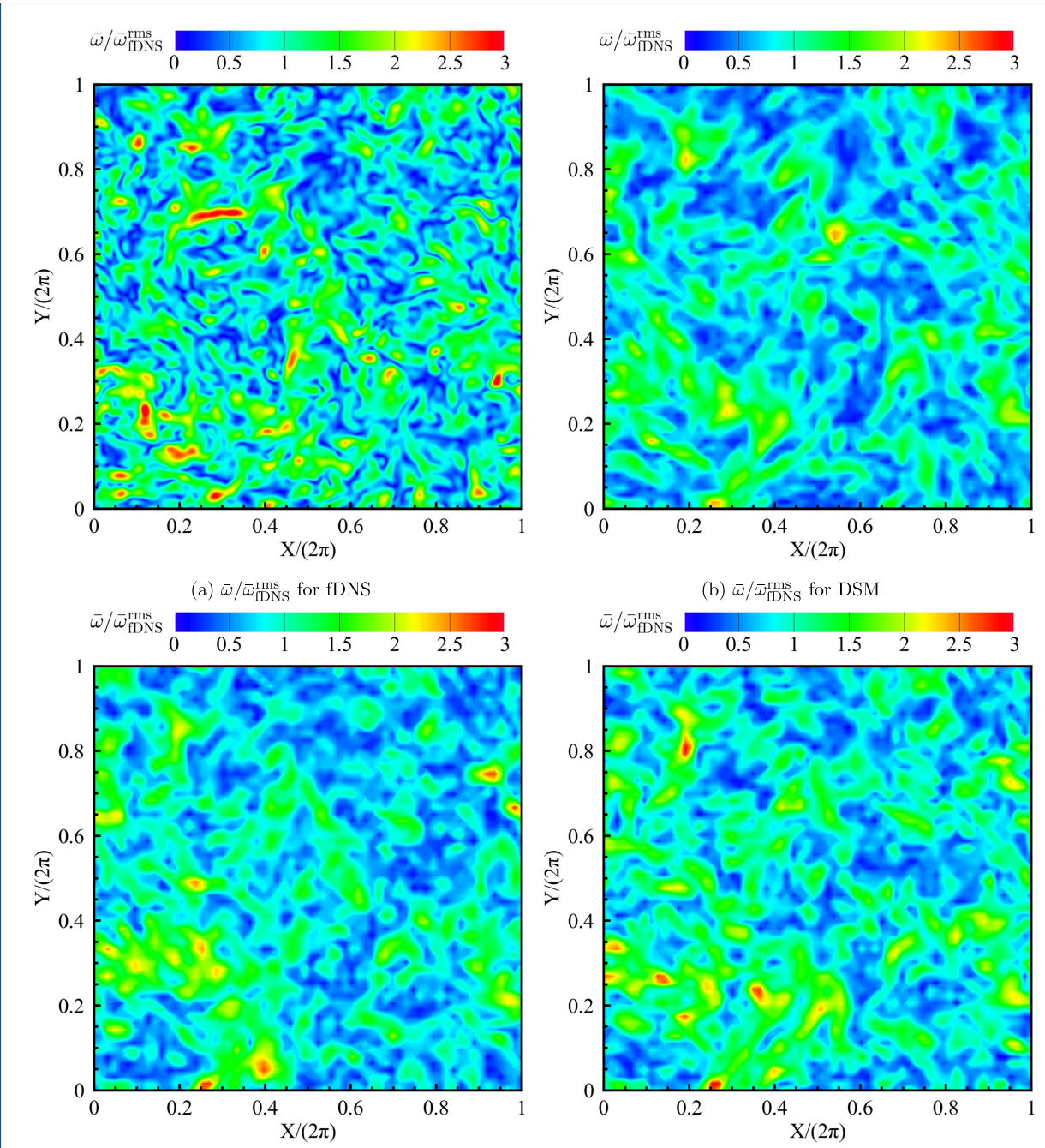

(c) $\bar{\omega} / \bar{\omega}_{\mathrm{fDNS}}^{\mathrm{rms}}$ for DNAM-GID

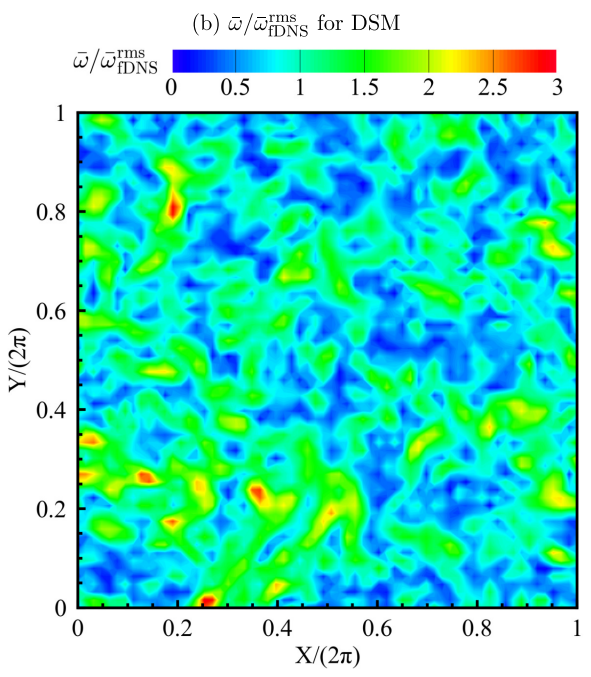

(d) $\bar{\omega} / \bar{\omega}_{\mathrm{fDNS}}^{\mathrm{rms}}$ for DNAM-SSD

Figure 11: Contours of the normalized vorticity magnitude at filter scale $\bar{\Delta}=$ $32 h_{\text {DNS }}$.

still holds, which are essential for the conventional dynamic models based on the Germano identity. The time step of LES is selected as $\Delta t_{\mathrm{LES}}=10 \Delta t_{\mathrm{DNS}}$ for all SGS models. The coarse-grained LES computations without any SGS models suffer from the numerical instability due to the nonlinear interactions between resolved large scales and residual scales. Implicit LES methods adopt the artificial dissipation to mimic the forward kinetic energy transfer from large scales to small scales. In this paper, we use the six-order compact-difference filtering scheme to provide necessary artificial dissipation for the numerical stability of coarse-grained LES computations, expressed as[56, 57]

$$
\alpha_{f} \check{f}_{i-1}+\check{f}_{i}+\alpha_{f} \check{f}_{i+1}=\sum_{n=0}^{3} \frac{a_{n}}{2}\left(f_{i+n}+f_{i-n}\right),
$$


where a check "' represents the dissipative filtering operation applied to the velocity field after each calculation of ILES, the parameter $0<\alpha_{f} \leq 0.5$, and four coefficients $a_{n}$ are determined by the Taylor-series expansion, namely[56, 57]

$$
a_{0}=\frac{11}{16}+\frac{5}{8} \alpha_{f}, \quad a_{1}=\frac{15}{32}+\frac{17}{16} \alpha_{f}, \quad a_{2}=\frac{-3}{16}+\frac{3}{8} \alpha_{f}, \quad a_{3}=\frac{1}{32}-\frac{1}{16} \alpha_{f} .
$$

In the paper, the control parameter $\alpha_{f}=0.495$ is chosen for a commonly-used sixorder low-dissipation filtering scheme[56, 57]. The pure scale-similarity type SGS model (DNAM-SSD) is numerically unstable due to lack of sufficient dissipation. Therefore, we couple the consistent compact-difference dissipative filtering scheme with the DNAM-SSD model to ensure the numerical stability of the LES calculations. The same instantaneous snapshot of the filtered DNS data is used as the initialization of the LES calculations for different SGS models. Table 5 summarizes the average computational time for the SGS stress modeling at both filter scales $\bar{\Delta}=16 h_{\mathrm{DNS}}$ and $32 h_{\mathrm{DNS}}$. Compared to the classical DSM and DMM models, the DNAM model would not particularly increase too much computational cost: the modeling time of the DNAM-GID model is about 1.1 times that of the DMM model. The proposed SSD procedure simplifies the calculation process of the conventional GID method, which greatly reduces the computation costs and only takes about half the time of the DNAM-GID model (0.6 times that of the DMM model) at both filter widths $\bar{\Delta}=16 h_{\mathrm{DNS}}$ and $32 h_{\mathrm{DNS}}$.

In the a posteriori testings of LES computations, we first compare the velocity spectra of different SGS models with those of the DNS and filtered DNS (fDNS) data at both filter scales $\bar{\Delta}=16 h_{\mathrm{DNS}}$ and $32 h_{\mathrm{DNS}}$ shown in Figures 5 and 6 . LES of the incompressible turbulence is governed by the filtered Navier-Stokes equations (Eqs. 6 and 7), therefore, the statistics of an ideal LES would be close to that of the fDNS data. The velocity spectra predicted by the ILES model are insufficiently dissipated and obviously higher than the fDNS data, while those modeled by the DSM and DMM models exhibit the tilde distribution due to excessive dissipation. For the DSM and DMM models, velocity spectra near the truncated wavenumbers are diminished by the model dissipation, resulting in the blockage of the kinetic energy cascade from large scales to small scales. Therefore the kinetic energy accumulates in the region of medium wavenumbers. The predicted velocity spectra of the DNAM-GID model are very similar to the DMM model, which has a significant deviation from the fDNS data. In contrast, the DNAM-SSD model outperforms the other SGS models and accurately reconstruct the velocity spectra at both filter scales $\bar{\Delta}=16 h_{\mathrm{DNS}}$ and $32 h_{\mathrm{DNS}}$.

The SGS energy flux characterizes the kinetic energy transfer between the resolved large scales and the unclosed subgrid scales. The positive SGS energy flux stands for the forward energy cascade, while the negative SGS energy flux represents the energy backscatters. The PDFs of the normalized SGS energy flux $\Pi / \epsilon_{\text {DNS }}$ at filter scales $\bar{\Delta}=16 h_{\mathrm{DNS}}$ and $32 h_{\mathrm{DNS}}$ are plotted in Figure 7 . The DSM model only reconstructs the forward SGS energy transfer whose modeled SGS energy flux is always non-negative. The PDFs of the SGS energy flux for the DMM and DNAMGID models are obviously narrower than the fDNS data. In comparison, the DNAMSSD model can accurately mimic both the forward SGS energy transfer and the energy backscatter. 
We further compare the normalized strain-rate tensor reconstructed by different SGS models, whose PDFs of the normal and shear components at both filter widths $\bar{\Delta}=16 h_{\mathrm{DNS}}$ and $32 h_{\mathrm{DNS}}$ are illustrated in Figures 8 and 9 , respectively. The strainrate tensor represents the local straining of the turbulence flow. ILES overestimates the strain-rate tensor and the predicted PDFs of the strain rate are obviously wider than the fDNS data, while those PDFs predicted by the DSM, DMM and DNAMGID models are apparently narrower due to the excessive model dissipation. In contrast, the DNAM-SSD model can recover more small scales and performs well on the predictions of the strain-rate tensor.

Figures 10 exhibits the PDFs of the normalized characteristic strain rate for different SGS models at filter scales $\bar{\Delta}=16 h_{\mathrm{DNS}}$ and $32 h_{\mathrm{DNS}}$. ILES obviously fails to predict the characteristic strain rate, while the DSM, DMM and DNAM-GID models cannot well capture the peak of the PDFs in reconstructing the characteristic strain rate. In comparison, the PDFs of the characteristic strain rate predicted by the DNAM-SSD model are very close to the fDNS data.

We finally examine the reconstruction ability of the turbulent coherent structure by comparing the transient contours of the normalized vorticity, shown in Fig. 11. The snapshots of LES calculations for different SGS models are selected on an arbitrary XY slice at the consistent time with approximately two large-eddy turnover periods. For the DSM and DNAM-GID models, some small-scale flow structures are excessively dissipated and only large scales are maintained. Compared to the other SGS models, the vortex structures reconstructed by the DNAM-SSD model exhibit more similar spatial distribution with the fDNS data, and more multiple-scale flow structures are accurately recovered by the proposed DNAM-SSD model.

\section{Conclusions}

In the current work, we develop a dynamic nonlinear algebraic model with the newly proposed scale-similarity dynamic procedure (DNAM-SSD) for large-eddy simulation of turbulence. In the DNAM-SSD model, the model coefficients are dynamically determined based on the scale-similarity relation, which greatly simplifies the conventional dynamic procedure based on the Germano identity (GID). The a priori analysis demonstrates that the proposed DNAM-SSD model outperforms the conventional velocity gradient model (VGM) and DNAM-GID model at a number of filter scales ranging from the inertial to dissipation ranges. The DNAM-SSD model gives the best prediction of the SGS stress with correlation coefficients higher than $95 \%$ and $92 \%$ as well as relative errors lower than $30 \%$ and $38 \%$ at corresponding filter scales $\bar{\Delta}=16 h_{\mathrm{DNS}}$ and $32 h_{\mathrm{DNS}}$ in comparison with the dynamic Smagorinsky model (DSM), dynamic mixed model (DMM), VGM model and DNAM-GID model, respectively. The proposed SSD procedure shows significant advantages over the conventional GID approach in determining the model coefficients of SGS models.

In the a posteriori testings of LES, the performance of the proposed DNAM-SSD model is examined at both filter widths $\bar{\Delta}=16 h_{\mathrm{DNS}}$ and $32 h_{\mathrm{DNS}}$. The classical implicit-LES (ILES), DSM, DMM and DNAM-GID models are used for comparisons of the a posteriori model accuracy. ILES fails to predict the statistics of turbulence with insufficient dissipation, while the DSM and DMM models are over-dissipative, leading to the fact that small scales are diminished by the excessive dissipation. 
The results predicted by the DNAM-GID model are very similar to those of the DMM model and have obvious deviations from the filtered DNS data. In contrast, the predictions of the DNAM-SSD model are very close to the filtered DNS data in the velocity spectra, the statistics of SGS energy flux and strain rate, as well as the instantaneous spatial structures of the vorticity magnitude at both filter scales without increasing the computational cost. The modeling time of the DNAMSSD model is only half the time of the DNAM-GID model (0.6 times that of the DMM model) at both filter widths. These results demonstrate that the current DNAM-SSD model is an effective framework for enhancing the advanced SGS stress modeling of LES.

\begin{abstract}
Acknowledgements
This work was supported by Center for Computational Science and Engineering of Southern University of Science and Technology.

Funding

This work was supported by the National Numerical Windtunnel Project (No. NNW2019ZT1-A04), by the National Natural Science Foundation of China (NSFC Grants No. 12172161, No. 91952104, No. 92052301, and No. 91752201), by the Shenzhen Science and Technology Program (Grants No. KQTD20180411143441009), by Key Special Project for Introduced Talents Team of Southern Marine Science and Engineering Guangdong Laboratory (Guangzhou) (Grant No. GML2019ZD0103), and by Department of Science and Technology of Guangdong Province (No. 2020B1212030001).
\end{abstract}

\title{
Availability of data and materials
}

The data that support the findings of this study are available from the corresponding author upon reasonable request.

\section{Competing interests}

The authors declare that they have no competing interests.

\section{Author details}

${ }^{1}$ Department of Mechanics and Aerospace Engineering, Southern University of Science and Technology, Shenzhen, 518055 China. ${ }^{2}$ Southern Marine Science and Engineering Guangdong Laboratory, Guangzhou, 511458 China. ${ }^{3}$ Guangdong-Hong Kong-Macao Joint Laboratory for Data-Driven Fluid Mechanics and Engineering Applications, Southern University of Science and Technology, Shenzhen, 518055 China. ${ }^{4}$ Department of Ocean Science, The Hong Kong University of Science and Technology, Hong Kong, 999077 China.

\section{References}

1. Pope, S.B.: Turbulent Flows. Cambridge University Press, Cambridge (2000)

2. Sagaut, P.: Large Eddy Simulation for Incompressible Flows: an Introduction, 3rd ed edn. Scientific computation. Springer, Berlin ; New York (2006)

3. Garnier, E., Adams, N., Sagaut, P.: Large Eddy Simulation for Compressible Flows. Scientific Computation. Springer, Dordrecht (2009)

4. Lesieur, M., Metais, O.: New Trends in Large-Eddy Simulations of Turbulence. Annu. Rev. Fluid Mech. 28(1), 45-82 (1996)

5. Meneveau, C., Katz, J.: Scale-Invariance and Turbulence Models for Large-Eddy Simulation. Annu. Rev. Fluid Mech. 32(1), 1-32 (2000). Publisher: Annual Reviews

6. Durbin, P.A.: Some Recent Developments in Turbulence Closure Modeling. Annu. Rev. Fluid Mech. 50(1), 77-103 (2018). Publisher: Annual Reviews

7. Moser, R.D., Haering, S.W., Yalla, G.R.: Statistical Properties of Subgrid-Scale Turbulence Models. Annu. Rev. Fluid Mech. 53(1), (2021)

8. Smagorinsky, J.: General circulation experiments with the primitive equations: I. the basic experiment. Mon. Wea. Rev. 91(3), 99-164 (1963)

9. Lilly, D.K.: The representation of small-scale turbulence in numerical simulation experiments. In: Proc. IBM Sci. Compt. Symp. Environ. Sci., White Plains, IBM, 1967 (1967)

10. Deardorff, J.W.: A numerical study of three-dimensional turbulent channel flow at large Reynolds numbers. J. Fluid Mech. 41(2), 453-480 (1970)

11. Clark, R.A., Ferziger, J.H., Reynolds, W.C.: Evaluation of subgrid-scale models using an accurately simulated turbulent flow. J. Fluid Mech. 91(01), 1 (1979)

12. Bardina, J., Ferziger, J., Reynolds, W.: Improved subgrid-scale models for large-eddy simulation. In: 13th Fluid and PlasmaDynamics Conference. American Institute of Aeronautics and Astronautics, Snowmass, CO,U.S.A. (1980)

13. Liu, S., Meneveau, C., Katz, J.: On the properties of similarity subgrid-scale models as deduced from measurements in a turbulent jet. J. Fluid Mech. 275, 83-119 (1994)

14. Boris, J.P., Grinstein, F.F., Oran, E.S., Kolbe, R.L.: New insights into large eddy simulation. Fluid Dyn Res 10(4), 199-228 (1992) 
15. Grinstein, F.F., Margolin, L.G., Rider, W.J.: Implicit Large Eddy Simulation: Computing Turbulent Fluid Dynamics vol. 113. Cambridge University Press, Cambridge (2007)

16. Xie, C., Wang, J., Li, H., Wan, M., Chen, S.: A modified optimal LES model for highly compressible isotropic turbulence. Phys. Fluids 30(6), 065108 (2018)

17. Chen, S., Xia, Z., Pei, S., Wang, J., Yang, Y., Xiao, Z., Shi, Y.: Reynolds-stress-constrained large-eddy simulation of wall-bounded turbulent flows. J. Fluid Mech. 703, 1-28 (2012)

18. Sarghini, F., de Felice, G., Santini, S.: Neural Networks Based Subgrid Scale Modeling in Large Eddy Simulations. Comput Fluids 32(1), 97-108 (2003)

19. Gamahara, M., Hattori, Y.: Searching for Turbulence Models by Artificial Neural Network. Phys. Rev. Fluids 2(5), 054604 (2017)

20. Xie, C., Wang, J., Li, K., Ma, C.: Artificial neural network approach to large-eddy simulation of compressible isotropic turbulence. Phys. Rev. E 99(5), 053113 (2019)

21. Yuan, Z., Xie, C., Wang, J.: Deconvolutional artificial neural network models for large eddy simulation of turbulence. Phys. Fluids 32(11), 115106 (2020)

22. Yuan, Z., Wang, Y., Xie, C., Wang, J.: Dynamic iterative approximate deconvolution models for large-eddy simulation of turbulence. Phys. Fluids 33(8), 085125 (2021)

23. Park, J., Choi, H.: Toward neural-network-based large eddy simulation: Application to turbulent channel flow. J. Fluid Mech. 914, 16 (2021)

24. Jiang, C., Vinuesa, R., Chen, R., Mi, J., Laima, S., Li, H.: An interpretable framework of data-driven turbulence modeling using deep neural networks. Phys. Fluids 33(5), 055133 (2021)

25. Subel, A., Chattopadhyay, A., Guan, Y., Hassanzadeh, P.: Data-driven subgrid-scale modeling of forced Burgers turbulence using deep learning with generalization to higher Reynolds numbers via transfer learning. Phys. Fluids 33(3), 031702 (2021)

26. Germano, M., Piomelli, U., Moin, P., Cabot, W.H.: A dynamic subgrid-scale eddy viscosity model. Phys. Fluids A: Fluid Dynamics 3(7), 1760-1765 (1991)

27. Lilly, D.K.: A proposed modification of the Germano subgrid-scale closure method. Phys. Fluids A: Fluid Dynamics 4(3), 633-635 (1992)

28. Zang, T.A., Dahlburg, R.B., Dahlburg, J.P.: Direct and large-eddy simulations of three-dimensional compressible Navier-Stokes turbulence. Phys. Fluids A: Fluid Dynamics 4(1), 127-140 (1992)

29. Vreman, B., Geurts, B., Kuerten, H.: On the formulation of the dynamic mixed subgrid-scale model. Phys. Fluids 6(12), 4057-4059 (1994)

30. Yu, C., Xiao, Z., Li, X.: Scale-adaptive subgrid-scale modelling for large-eddy simulation of turbulent flows. Phys. Fluids 29(3), 035101 (2017)

31. Zhou, Z., Wang, S., Yang, X., Jin, G.: A structural subgrid-scale model for the collision-related statistics of inertial particles in large-eddy simulations of isotropic turbulent flows. Phys. Fluids 32(9), 095103 (2020)

32. Vreman, B., Geurts, B., Kuerten, H.: Large-eddy simulation of the turbulent mixing layer. J. Fluid Mech. 339 , 357-390 (1997)

33. Ghosal, S., Lund, T.S., Moin, P., Akselvoll, K.: A dynamic localization model for large-eddy simulation of turbulent flows. J. Fluid Mech. 286, 229-255 (1995)

34. Pope, S.B.: A more general effective-viscosity hypothesis. J. Fluid Mech. 72(2), 331-340 (1975)

35. Lund, T.S., Novikov, E.A.: Parameterization of Subgrid-Scale Stress By the Velocity Gradient Tensor. Cent. Turbul. Res. Stanf. Univ. NASA (1992)

36. Speziale, C.G.: Analytical Methods for the Development of Reynolds-Stress Closures in Turbulence. Annu. Rev. Fluid Mech. 23(1), 107-157 (1991)

37. Gatski, T.B., Speziale, C.G.: On explicit algebraic stress models for complex turbulent flows. J. Fluid Mech. 254, 59-78 (1993)

38. Wong, V.C.: A proposed statistical-dynamic closure method for the linear or nonlinear subgrid-scale stresses. Phys. Fluids Fluid Dyn. 4(5), 1080-1082 (1992)

39. Kosović, B.: Subgrid-scale modelling for the large-eddy simulation of high-Reynolds-number boundary layers. J. Fluid Mech. 336, 151-182 (1997)

40. Wang, B.-C., Bergstrom, D.J.: A dynamic nonlinear subgrid-scale stress model. Physics of Fluids 17(3), 035109 (2005)

41. Wang, B.-C., Yee, E., Bergstrom, D.J., lida, O.: New dynamic subgrid-scale heat flux models for large-eddy simulation of thermal convection based on the general gradient diffusion hypothesis. J. Fluid Mech. 604, 125-163 (2008)

42. Marstorp, L., Brethouwer, G., Grundestam, O., Johansson, A.V.: Explicit algebraic subgrid stress models with application to rotating channel flow. J. Fluid Mech. 639, 403-432 (2009)

43. Rasam, A., Brethouwer, G., Johansson, A.V.: A stochastic extension of the explicit algebraic subgrid-scale models. Phys. Fluids 26(5), 055113 (2014)

44. Xie, C., Yuan, Z., Wang, J.: Artificial neural network-based nonlinear algebraic models for large eddy simulation of turbulence. Phys. Fluids 32(11), 115101 (2020)

45. Wang, Y., Yuan, Z., Xie, C., Wang, J.: Artificial neural network-based spatial gradient models for large-eddy simulation of turbulence. AIP Adv. 11(5), 055216 (2021)

46. Wang, Y., Zelong, Y., Xie, C., Wang, J.: A dynamic spatial gradient model for the subgrid closure in large-eddy simulation of turbulence. Phys. Fluids 33, 075119 (2021)

47. Wang, J., Shi, Y., Wang, L.-P., Xiao, Z., He, X.T., Chen, S.: Effect of compressibility on the small-scale structures in isotropic turbulence. J. Fluid Mech. 713, 588-631 (2012)

48. Wang, J., Wan, M., Chen, S., Xie, C., Zheng, Q., Wang, L.-P., Chen, S.: Effect of flow topology on the kinetic energy flux in compressible isotropic turbulence. J. Fluid Mech. 883, 11 (2020)

49. Shi, Y., Xiao, Z., Chen, S.: Constrained subgrid-scale stress model for large eddy simulation. Phys. Fluids 20(1), 011701 (2008)

50. Chen, S., Doolen, G.D., Kraichnan, R.H., She, Z.-S.: On statistical correlations between velocity increments and 
locally averaged dissipation in homogeneous turbulence. Phys. Fluids A: Fluid Dynamics 5(2), 458-463 (1993)

51. Ishihara, T., Kaneda, Y., Yokokawa, M., Itakura, K., Uno, A.: Small-scale statistics in high-resolution direct numerical simulation of turbulence: Reynolds number dependence of one-point velocity gradient statistics. J. Fluid Mech. 592, 335-366 (2007)

52. Ishihara, T., Gotoh, T., Kaneda, Y.: Study of High-Reynolds Number Isotropic Turbulence by Direct Numerical Simulation. Annu. Rev. Fluid Mech. 41(1), 165-180 (2009)

53. Chow, F.K., Moin, P.: A further study of numerical errors in large-eddy simulations. J. Comput. Phys. 184(2), 366-380 (2003)

54. Xie, C., Wang, J., E, W.: Modeling Subgrid-Scale Forces by Spatial Artificial Neural Networks in Large Eddy Simulation of Turbulence. Phys. Rev. Fluids 5(5), 054606 (2020)

55. Yang, X.I.A., Griffin, K.P.: Grid-point and time-step requirements for direct numerical simulation and large-eddy simulation. Phys. Fluids 33(1), 015108 (2021)

56. Visbal, M.R., Gaitonde, D.V.: On the Use of Higher-Order Finite-Difference Schemes on Curvilinear and Deforming Meshes. J. Comput. Phys. 181(1), 155-185 (2002)

57. Visbal, M.R., Rizzetta, D.P.: Large-Eddy Simulation on Curvilinear Grids Using Compact Differencing and Filtering Schemes. J. Fluids Eng. 124(4), 836-847 (2002) 